\title{
Conocimientos, actitudes y prácticas del paciente hipertenso
}

\section{Health knowledge, attitudes and practices of hypertensive patients}

\author{
César Giovanni Gómez Cuéllar', Beatriz Magola Orozco Coneo', Manuel Alejandro \\ Suárez Camargo', Alexander Rivera Cuellar', Dolly Castro Betancourth²
}

\begin{abstract}
Resumen
Objetivo: Describir los conocimientos, actitudes y prácticas adoptadas por los pacientes hipertensos, frente a su enfermedad y al régimen terapéutico, en la Ciudad de Florencia. Método: Estudio descriptivo transversal cuantitativo. La muestra fue de $\mathbf{2 5 0}$ pacientes con hipertensión arterial, seleccionados mediante muestreo probabilístico sistemático. Resultados: El $80 \%$ de los pacientes correspondieron al sexo femenino con una razón de 4 mujeres por cada hombre encuestado. La edad mediana fue de 64 años. El $71,86 \%$ tenían conocimiento de la enfermedad y el régimen terapéutico; el $69,64 \%$ de los pacientes hipertensos presentaron una actitud positiva y $60,95 \%$ presentaron prácticas positivas en el manejo de la hipertensión arterial y el régimen terapéutico. Conclusiones: Un alto porcentaje de los pacientes hipertensos tuvieron conocimiento de la enfermedad y del régimen terapéutico, principalmente en lo relacionado con la dieta, complicaciones y el régimen farmacológico. Los pacientes presentaron una actitud positiva frente a la enfermedad y al régimen terapéutico, reconociendo la enfermedad como amenazante para la salud y a la atención brindada por el personal de salud, como positiva para la misma. En cuanto a las prácticas se encontró que presentan prácticas positivas principalmente en el cumplimiento en las citas de control, identificación de signos de alarma y consideran que la información que reciben del personal de salud es suficiente para comprender el tratamiento. Los pacientes presentan prácticas hacia la utilización de remedios caseros para el manejo de la hipertensión arterial.
\end{abstract}

Palabras clave: Hipertensión; Conocimientos; Actitudes; Prácticas.

\begin{abstract}
Objective: Give an account of the health knowledge, attitudes and practices adopted by hypertensive patients in Florencia in terms of their disease and therapeutic regimen. Method: A descriptive, transversal and quantitative study. The sample consisted of 250 patients with high blood pressure, selected using systematic probability sampling. Results: $80 \%$ of patients were female, with four women studied for every man. The average age was $64.71 .86 \%$ of patients were familiar with the disease and the therapeutic regimen; $69.64 \%$ of the hypertensive patients showed a positive attitude and $60.95 \%$ demonstrated good practices in managing high blood pressure and the therapeutic regimen. Conclusions: A high percentage of hypertensive patients were familiar with the disease and the therapeutic regimen, primarily with regard to diet, complications and the medication regimen. Patients showed a positive attitude towards the disease and the therapeutic regimen, recognising that the disease is a serious threat to health and that the care provided by health care professionals has a positive effect. As for the practices of the patients, it was found that positive practices were shown principally by attending follow-up appointments and identifying warning signs and it was considered that the information provided by the health care professionals was sufficient in order to understand their treatment. The patients also used home remedies in order to manage high blood pressure.
\end{abstract}

Key words: Hypertension; Health Knowledge; Attitudes; Practice

1. Especialista en Epidemiologia Universidad Surcolombiana

2. Docente Tiempo Completo, Universidad Surcolombiana

Correspondencia: Alexander Rivera Cuéllar. Correo electrónico: alricu2006@hotmail.com

Recibido: 11/06/2014 Revisado: 17/12/2014 Aceptado: 01/06/2015 


\section{Introducción}

Por su alta morbilidad-mortalidad la Hipertensión Arterial (HTA), es problema en salud pública en el mundo y en nuestro país, siendo el factor de riesgo para enfermedades crónicas y degenerativas de alto costo como la enfermedad cerebrovascular, la enfermedad coronaria, enfermedad arterioesclerótica y enfermedad renal.

La HTA exige el cumplimiento del tratamiento de forma permanente y sistemática, e implica cambios en el estilo de vida combinados o no con la toma de fármacos. En el caso de la HTA, por adherencia al tratamiento o seguimiento terapéutico, se entiende la incorporación de nuevos hábitos en las rutinas de la vida del paciente y cumplimiento del plan farmacológico. A pesar de existir un tratamiento eficaz, la mitad de los pacientes tratados por HTA, abandonan por completo la atención a partir del primer año del diagnóstico $\mathrm{y}$, de los que permanecen bajo supervisión médica, solo $50 \%$ toma al menos el $80 \%$ de sus medicamentos prescritos y debido a la deficiente adherencia al tratamiento antihipertensor, aproximadamente un $75 \%$ de los pacientes con diagnóstico de HTA no logran el control óptimo de la presión arterial ${ }^{1}$.

Uno de los problemas más críticos que enfrentan médicos, personal de enfermería, pacientes y prácticamente todo el sistema de salud es "la falta de adherencia o incumplimiento" del tratamiento, esto incluye los cambios del estilo de vida (dieta, ejercicio físico, abandono de los hábitos de vida nocivos, entre otros) y el tratamiento farmacológico ${ }^{2}$.

La identificación de factores que modulan la adherencia terapéutica supone el primer paso, para posteriormente, elaborar estrategias que faciliten o mejoren su mantenimiento. Dichas estrategias deben estar encaminadas a la educación del paciente hipertenso, considerada como la piedra angular de los pilares del tratamiento, que durará toda la vida y responderá cada vez más a las etapas por las que atraviese el paciente $^{3}$.

Teniendo en cuenta que las enfermedades hipertensivas ocuparon el cuarto lugar de mortalidad en mayores de 65 años (tasa 242.6/100.000 habitantes) en la población colombiana ${ }^{4}$ y que en el departamento del Caquetá, de acuerdo con la encuesta Nacional de Salud 2007, el $11,1 \%$ de la población de 18 a 69 años, refirió haber tenido diagnóstico médico de HTA alguna vez (11,5\% promedio nacional $)^{5}$, se hace necesario realizar estudios a nivel local y regional, como antecedente investigativo, basado en la no existencia de estudios relacionados con el tema, con el fin de reorientar las acciones que en salud pública se llevan a cabo,

Esta investigación se llevó a cabo con pacientes diagnosticados con HTA, en la ESE Hospital Comunal Las Malvinas, Institución Prestadora de Servicios de Salud de baja complejidad, de carácter público del Municipio de Florencia del Departamento del Caquetá, durante los meses de noviembre 2013 a enero del año 2014.

\section{Materiales y métodos}

Diseño: El estudio correspondió a un estudio descriptivo de tipo transversal donde la recolección de la información se recolecto de noviembre de 2013 a enero de 2014.

Población: La población correspondió a 4500 pacientes diagnosticados con HTA del programa de Crónicos de la E.S.E Hospital Comunal Malvinas, pertenecientes a los regímenes subsidiado, contributivo, excepción y pobres no asegurados, del área urbana y rural del municipio de Florencia Caquetá. Selección de la muestra: La muestra se calculó con el programa EPI INFO versión 3.5.1; con nivel de confianza del $95 \%$, una frecuencia esperada del $20 \%$ y con error estimado del $5 \%$, el tamaño de la muestra fue de 250 pacientes con HTA de la E.S.E Hospital Comunal Malvinas. El criterio de selección de la muestra se llevó a cabo por medio de muestreo probabilístico sistemático.

Métodos para obtener la información: La técnica que se utilizó para la recolección de los datos fue a través de la aplicación de un instrumento dirigido por el grupo investigador.

Instrumento: $\mathrm{El}$ instrumento constó de cuatro partes: la primera contenía cinco ítems de selección múltiple con única respuesta, para determinar las características sociodemográficas. La segunda parte contenía doce (12) enunciados que permitieron evaluarlos conocimientos, de los participantes frente a la enfermedad y el régimen terapéutico. La tercera parte constaba de un cuestionario tipo Likert, que permitió determinar las actitudes a través de once (11) enunciados, con opciones de respuesta o categorías de: Totalmente de acuerdo, De acuerdo, Ni de acuerdo ni en desacuerdo, En desacuerdo, Totalmente en desacuerdo. La cuarta parte permitió evaluar las prácticas del paciente, por medio de doce (12) enunciados con opciones de respuesta de: siempre, Casi siempre, A veces, Casi nunca y Nunca. La validez del instrumento se realizó mediante una revisión por expertos. Hernández Sampieri ${ }^{6}$, considera que la revisión por expertos se refiere al grado en que aparentemente un instrumento de medición mide la variable en cuestión, de acuerdo con "voces calificadas", se encuentra vinculado a la validez de contenido.

Análisis estadístico: Después de la recolección de la información, se procedió a codificarla, tabularla y analizarla haciendo uso del programa Microsoft Excel 2007 y del programa estadístico SSPS versión 14.0 para Windows versión gratuita para estudiantes. Se realizó análisis descriptivo, a través de análisis bivariado y básico, que permitió obtener porcentajes y frecuencias. Se aplicaron pruebas de significancia estadística como T Student y de Chi cuadro

En el desarrollo de la investigación se tuvo en cuenta los parámetros establecidos en la Declaración de Helsinki, las Normas de Buenas Prácticas Clínicas, las Pautas Éticas Internacionales para la Experimentación Biomédica en Seres Humanos y la Resolución No 008430 de 1993. Además se contó con el concepto del comité técnico científico de la ESE Hospital Comunal Malvinas, y por el Comité de Ética de la Facultad de Salud, de la Universidad Surcolombiana, quienes dieron el aval, de acuerdo a la reglamentación establecida para el desarrollo de investigación en salud. 
R.F.S Revista Facultad de Salud

Enero-Junio de 2015;7(1): 32-38

\section{Resultados}

\section{Caracteristicas sociodemográficas}

Las principales características sociodemográficas son presentadas en la Tabla 1.

\section{Conocimientos, actitudes y prácticas}

Los resultados indicaron que de los pacientes encuestados el 71,86\% tenían conocimiento de la enfermedad y del régimen terapéutico. Se encontró que la mayoría de los pacientes conocían de la dieta que deben llevar los pacientes

Tabla 1. Características de los pacientes hipertensos, ESE Hospital Comunal Malvinas, Florencia, 2013-2014.

\begin{tabular}{lcc}
\hline Característica & Frecuencia & $\%$ \\
\hline Sexo & & \\
Masculino & 50 & 20 \\
Femenino & 200 & 80 \\
\hline Edad & & \\
$20-29$ & 1 & 0,4 \\
$30-39$ & 4 & 1,6 \\
$40-49$ & 23 & 9,2 \\
$50-59$ & 62 & 24,8 \\
$60-69$ & 75 & 30 \\
$70-79$ & 57 & 22,8 \\
$80-89$ & 26 & 10,4 \\
$90-99$ & 2 & 0,8 \\
\hline Estado civil & & \\
Casado & 75 & 30,0 \\
Separado & 23 & 9,2 \\
Soltero & 61 & 24,4 \\
Unión libre & 39 & 15,6 \\
Viudo & 52 & 20,8 \\
\hline
\end{tabular}

\section{Nivel educativo}

Básica primaria completa

Básica primaria incompleta

\section{1}

92

Secundaria completa

Secundaria incompleta

Educación técnica

Sin formación académica

\section{Estrato socioeconómico}

1

2

3

\section{Ocupación}

Hogar

Sin trabajo

Trabajador
20,4

36,8

5,6

10,8

0,4

26 hipertensos, de sus complicaciones, así como del control farmacológico de la enfermedad. Sin embargo, se presentó desconocimiento en relación a las cifras normales de tensión arterial, la definición HTA y la suspensión del tratamiento cuando se presentan cifras normales de tensión arterial (Tabla 2).

Para consolidar la información sobre las actitudes, se consideraron las respuestas totalmente en desacuerdo y en desacuerdo como actitudes negativas; las respuestas ni de acuerdo ni en desacuerdo como neutras y las respuestas de acuerdo y totalmente de acuerdo como actitudes positivas. Los resultados mostraron que el 69,64\% de los pacientes hipertensos presentaron una actitud positiva, el $16,44 \%$ presentaron actitud negativa y el $13,92 \%$ presentaron una actitud neutra frente a la enfermedad y al régimen terapéutico. El 94,4\% de los pacientes consideraron la enfermedad como una amenaza para la salud, y piensan que la atención que reciben del personal de salud ha contribuido para mejorar su estado de salud, asumiendo la HTA como una enfermedad para toda la vida, que implica sufrir con resignación. Se presentaron actitudes negativas, ya que el $36 \%$ manifestó haber dejado de tomar los medicamentos de vez en cuando, el 30,4\% emplearon remedios naturales para controlar la HTA, considerando que son más seguros que los medicamentos, y el 21,6\% creen que el mejor momento para tratar la enfermedad es cuando se sienten mal (Tabla 3).

Para consolidar la información sobre las prácticas se consideraron las respuestas nunca o casi nunca, como practicas negativa; las respuestas a veces como neutras y las respuestas casi siempre y siempre como practicas positivas. Los resultados mostraron que los pacientes hipertensos presentaron $60,95 \%$ prácticas positivas en el manejo de la hipertensión arterial y el régimen terapéutico, el 30,46\% tuvieron prácticas negativas y el $8,58 \%$ realizaban prácticas neutras. El $98,4 \%$ de los pacientes presentaron prácticas positivas al cumplir con las citas de control y seguimiento programadas, el 98,8\% ante signos de alarma asistían de forma inmediata al centro de salud y/o hospital y el 97,6\% manifestó que la información que recibieron del personal de salud suficiente para comprender el tratamiento. Dentro de las practicas negativas se halló, el uso de remedios caseros en un $48,2 \%$ y el reemplazo del tratamiento farmacológico por este en un 47,6\% (Tabla 4).

Análisis bivariado: Se encontró que no existen diferencias estadísticamente significativas entre los promedios de edad, y el conocimiento de una dieta baja en grasa y sal como fundamental para el control de la tensión arterial. Con un nivel de confiabilidad de $95 \%$ con un valor de $\mathrm{p}=0,890$; además de acuerdo con un Chi cuadrado de 0.41 y un nivel crítico de 0.623 . De igual manera no se encontró relación significativamente estadística, entre la edad y el conocimiento de que la presión arterial mal controlada puede ocasionar problemas en el corazón, cerebro, riñón y los ojos, con un nivel de confiabilidad de $95 \%$ y un valor de $\mathrm{p}=0,403$; con un Chi cuadrado de Pearson de 0.601 y valor crítico o significancia asintótica de 0.438 . 
Tabla 2. Porcentaje de respuesta por pregunta de la variable conocimientos.

\begin{tabular}{|c|c|c|c|}
\hline$N^{\circ}$ & Conocimientos & $\%$ & \\
\hline & Enunciado & sí & NO \\
\hline 1 & La hipertensión arterial es el aumento de la tensión por encima de 120/80 mmHg & 74 & 26 \\
\hline 2 & La hipertensión arterial es una enfermedad para toda la vida. & 83,2 & 16,8 \\
\hline 3 & Las cifras de tensión arterial por debajo de $140 / 90 \mathrm{mmHg}$ se consideran normales & 16,8 & 83,2 \\
\hline 4 & Una dieta baja grasa y sal es fundamental para el control de la tensión arterial. & 97,2 & 2,8 \\
\hline 5 & El consumo de cigarrillo, alcohol aumenta la presión arterial & 93,6 & 6,4 \\
\hline 6 & La tensión alta trae complicaciones al cerebro, corazón, riñón & 96,8 & 3,2 \\
\hline 7 & $\begin{array}{l}\text { Se puede suspender el tratamiento cuando las cifras de presión arterial se encuentren dentro } \\
\text { de límites normales. }\end{array}$ & 40,4 & 59,6 \\
\hline 8 & $\begin{array}{l}\text { La presión arterial mal controlada puede ocasionar problemas en el corazón, cerebro, } \\
\text { riñón y los ojos. }\end{array}$ & 96,0 & 4,0 \\
\hline 9 & $\begin{array}{l}\text { Cuando sube la tensión arterial aparecen síntomas como dolor de cabeza, ruidos en los oídos, } \\
\text { palpitaciones, dolor en el pecho, calor en la cara. }\end{array}$ & 88,4 & 11,6 \\
\hline 10 & La hipertensión arterial se puede controlar con medicamentos & 94,4 & 5,6 \\
\hline 11 & $\begin{array}{l}\text { Se puede aumentar o disminuir las dosis y horario de los medicamentos, si controla su tensión } \\
\text { arterial, sin consultar con el personal de salud. }\end{array}$ & 46,8 & 53,2 \\
\hline 12 & Si la persona es hipertensa y se siente bien, es necesario acudir al médico. & 82,8 & 17,2 \\
\hline
\end{tabular}

Tabla 3. Valoración de la actitud de los pacientes hipertensos frente a su enfermedad y el régimen terapéutico.

\begin{tabular}{|c|c|c|c|}
\hline Enunciado & $\%$ negativa & $\%$ neutra & $\%$ positiva \\
\hline $\begin{array}{l}\text { El tratamiento con medicamentos podemos interrumpirlo cuando la } \\
\text { hipertensión arterial está controlada }\end{array}$ & 21,6 & 6,8 & 71,6 \\
\hline $\begin{array}{l}\text { La hipertensión arterial es una enfermedad para toda la vida, e implica } \\
\text { sufrir con resignación }\end{array}$ & 12 & 8,4 & 79,6 \\
\hline La hipertensión es una enfermedad que amenaza la salud. & 1,2 & 4,4 & 94,4 \\
\hline $\begin{array}{l}\text { Para tratar la hipertensión arterial cree usted que es mejor usar los remedios } \\
\text { caseros, que le han recomendado un amigo, yerbatero o chaman }\end{array}$ & 24 & 3,2 & 72,8 \\
\hline $\begin{array}{l}\text { Los hipertensos deberían dejar los medicamentos durante algún tiempo } \\
\text { de vez en cuando. }\end{array}$ & 36 & 9,6 & 54,4 \\
\hline $\begin{array}{l}\text { Los remedios naturales para controlar la tensión arterial son más seguros } \\
\text { que los medicamentos. }\end{array}$ & 30,4 & 7,2 & 62,4 \\
\hline $\begin{array}{l}\text { Si el personal de salud dedicara más tiempo para el paciente, recetarían } \\
\text { menos medicamentos. }\end{array}$ & 2,4 & 89,2 & 8,4 \\
\hline $\begin{array}{l}\text { La atención que recibe del personal de salud ha contribuido para mejorar } \\
\text { su salud. }\end{array}$ & 4 & 2 & 94 \\
\hline El mejor momento para tratar su enfermedad es cuando se siente mal. & 28 & 5,2 & 66,8 \\
\hline $\begin{array}{l}\text { El tratamiento y las recomendaciones instauradas por el personal de salud } \\
\text { es el adecuado para el mejoramiento de su salud. }\end{array}$ & 4,8 & 3,2 & 92 \\
\hline Promedio & 16,44 & 13,92 & 69,64 \\
\hline
\end{tabular}


Tabla 4. Prácticas de los pacientes hipertensos frente a su enfermedad y el régimen terapéutico.

\begin{tabular}{|c|c|c|c|}
\hline Enunciado & $\%$ negativa & $\%$ neutra & $\%$ positiva \\
\hline Cumple con las citas de control y seguimiento programadas. & 0 & 1,6 & 98,4 \\
\hline Toma los medicamentos en el horario establecido por el personal de salud. & 7,2 & 9,2 & 83,6 \\
\hline $\begin{array}{l}\text { Si presenta dolor de cabeza, visión borrosa, dolor en el pecho, calor en la } \\
\text { cara, usted asiste de forma inmediata a un centro de salud y/o Hospital. }\end{array}$ & 0 & 1,2 & 98,8 \\
\hline Toma los medicamentos de acuerdo a las dosis indicadas por su médico. & 29,2 & 8,4 & 62,4 \\
\hline Usted utiliza remedios caseros para controlar su tensión arterial. & 30,8 & 20,8 & 48,4 \\
\hline Usted ha reemplazado el tratamiento farmacológico por remedios caseros. & 28,4 & 24 & 47,6 \\
\hline Usted practica ejercicio físico. & 2,8 & 8,8 & 88,4 \\
\hline La dieta que usted consume es baja en sal, grasa y condimentos. & 97,2 & 2 & 0,8 \\
\hline Consume usted bebidas alcohólicas. & 98 & 1,2 & 0,8 \\
\hline Consume usted cigarrillo, tabaco y/o sustancias. & 17,2 & 24,4 & 58,4 \\
\hline $\begin{array}{l}\text { Si usted presenta elevación de la tensión arterial, dolor de cabeza intenso, } \\
\text { visión borrosa, usted espera hasta sentirse bien antes de ir al médico }\end{array}$ & 82 & 2,4 & 15,6 \\
\hline $\begin{array}{l}\text { Usted consume medicamentos para controlar su tensión arterial no formulados } \\
\text { por personal de salud. }\end{array}$ & 3,2 & 5,2 & 91,6 \\
\hline $\begin{array}{l}\text { La información que recibe del personal de salud es suficiente para comprender } \\
\text { el tratamiento }\end{array}$ & 0 & 2,4 & 97,6 \\
\hline Promedio & 30,46 & 8,58 & 60,95 \\
\hline
\end{tabular}

\section{Discusión}

Doscientos cincuenta pacientes hipertensos de la ESE Hospital Comunal Malvinas de la Ciudad de Florencia, principalmente mujeres, de diferentes edades, estrato socioeconómico, nivel educativo dieron respuesta satisfactoria a la encuesta en donde se indagó por los conocimientos, actitudes y prácticas de los pacientes hipertensos.

La mayoría de pacientes poseían información acerca de la definición, del tratamiento, de las complicaciones y estilos de vida saludables. Estos resultados discrepan de los estudios de $\mathrm{Acosta}^{3}$ y Buendía ${ }^{7}$ en donde el 57,6\% de los pacientes desconocían los factores de riesgo y el $40 \%$ desconocían los órganos que podía afectar, respectivamente. Los pacientes poseen un mayor conocimiento en la dieta recomendada, las complicaciones que se generan de la HTA y conocen la efectividad terapéutica de los medicamentos para el control de la tensión arterial.

Adicionalmente los pacientes hipertensos tienen desconocimiento sobre las cifras normales de tensión arterial, y la definición de HTA, además el 59,6\% creen que se puede suspender el tratamiento cuando se tiene cifras tensionales dentro de los límites normales. Esto indica que los pacientes deben ser estimulados a reconocer la enfermedad claramente, los factores de riesgo y el régimen terapéutico como la principal medida en el manejo de los pacientes para el reconocimiento de la enfermedad y manejo de la misma ${ }^{8}$

$\mathrm{Al}$ analizar la categoría actitudes, se obtuvo un porcentaje alto positiva frente a la enfermedad y el régimen terapéutico. Lo anterior podría indicar un factor protector frente a la enfermedad. Pues como lo indica Huertas", "las actitudes son y están prediciendo las conductas y, si se desea cambiar una conducta, es necesario cambiar la actitud". De ahí la importancia de cuantos elementos se dan en la ante sala del comportamiento.

Los hipertensos presentaron una mayor actitud positiva reconociendo la enfermedad como una amenaza para la salud. Esto se indica como algo positivo ya que una persona sólo aceptará un cambio de estilo de vida o una restricción impuesta por un tratamiento si es capaz de percibir las posibilidades de riesgos que implicaría el no asumir este cambio de vida o restricción ${ }^{10}$. Además reconocen que la atención que 
reciben del personal de salud ha contribuido para mejorar su estado de salud; lo cual comparando estos resultados con los obtenidos en la investigación de $A_{\operatorname{costa}}{ }^{3}$ y Buendía ${ }^{7}$, se observó discrepancias ya que el $45,4 \%$ planteó recibir los beneficios parcialmente y al 3\% le resultó indiferente el tratamiento y el alrededor del $48 \%$ de los pacientes refiere olvidar en algún momento tomar el medicamento antihipertensivo.

Por otra parte existe un porcentaje alto de pacientes que consideran que la enfermedad es para toda la vida y que deben sufrir con resignación, lo que indicaría algún grado de desmotivación frente a la enfermedad, se convertiría en un riesgo para el paciente hipertenso, ya que la motivación es lo que mueve a actuar a la persona. Lo que pone su conducta en marcha, por tanto podría tener menos interés por los cambios de estilos de vida, cumplir con el régimen terapéutico, lo que llevaría mayores complicaciones de la enfermedad, es decir es una actitud negativa para el paciente hipertenso, ya que aunque es una enfermedad crónica no se trata de sufrir con resignación, pues los pacientes con hipertensión arterial con un tratamiento adecuado y con actitud positiva frente a la enfermedad pueden tener una calidad de vida normal. Además se encontró como actitudes negativas en los pacientes la creencia de que los remedios caseros sean más efectivos que los medicamentos, dejar los medicamentos durante algún tiempo y tratar la enfermedad cuando presentan alteraciones mayores; por lo cual es necesario tener en cuenta que los pacientes se deben identificar los tratamientos tradicionales que contribuyen al manejo de la enfermedad y concienciar de los riesgos y las complicaciones dejar el tratamiento o no consultar ante signos de alarma tempranamente.

De las prácticas que realizan los pacientes hipertensos, se encuentran el cumplimiento de las citas de control programadas, la identificación de signos de alarma y consideran que la información que reciben del personal de salud es suficiente, contraria al estudio de Buendía, en donde el $68 \%$ considera que si los médicos tuvieran más tiempo para los pacientes, recetarían menos medicamentos. Las practicas mencionadas anteriormente, se convierten en factores protectores para los pacientes hipertensos, pues al cumplir con las citas de control, su seguimiento será efectivo, además que reconoce y asiste ante la presencia de signos de alarma, disminuyendo el riesgo de complicaciones y la muerte. Sin olvidar que las recomendaciones que les brinda el personal de salud son atendidas y suficientes.

Se encontró en el estudio que los pacientes hipertensos, realizan prácticas a través del uso de los medicamentos tradicionales, ya que utilizan tratamientos caseros para controlar la tensión arterial y han suspendido el tratamiento farmacológico por remedios caseros, esto se puede deber a la interferencia que ejercen los patrones culturales en las poblaciones, lo cual ocasiona el choque entre el saber popular y las medidas aprobadas para el manejo de la enfermedad; de allí la importancia de realizar estrategias conjuntas con la comunidad, para que la población reconozca el manejo de la enfermedad y que las prácticas culturales no interfieran negativamente.
Se obtuvo que los pacientes con un nivel de básica primaria incompleta, que fueron la mayoría, consumen medicamentos no formulados por el personal de salud, observándose que podría estar relacionado el nivel educativo con esta práctica, la cual constituye en un riesgo para la salud.

En cuanto al conocimiento de una dieta adecuada y el rango de edad de los pacientes hipertensos, se evidenció que el $62.6 \%$ de los pacientes entre los 53 y los 72 años afirman que si saben que una dieta baja en grasa y sal, es fundamental para el control de la tensión arterial. Por el contrario el 42.9\% de las personas entre 73 y 82 años afirman no saber que una dieta baja en grasa y sal es fundamental para el control de la tensión arterial. Este resultado no fue significativamente estadístico (Chi Cuadrado de 0,241 y p =0,623).

Existe un gran porcentaje de paciente hipertensos entre los 63 y 72 años que no reconocen que la presión arterial mal controlada puede ocasionar problemas en órganos blanco como corazón, cerebro, riñón y los ojos. Por lo tanto es importante que los pacientes reconozcan estas complicaciones, y que puedan identificar signos de alarma, para que consulten a tiempo y de esta manera disminuyen las incapacidades, la morbilidad y mortalidad por complicaciones. Los resultados obtenidos señalan que no existe relación entre estas dos variables (Chi Cuadrado de 0,601 y $\mathrm{p}=0,438)$.

\section{Agradecimientos}

Los autores expresan sus agradecimientos a la Universidad Surcolombiana y Especialización en Epidemiología, por el conocimiento aportado durante la formación y la investigación.

A Dolly Castro Betancourt, Magister en Salud Pública y Epidemiología, por transmitirnos sus conocimientos, experiencia investigativa y orientación en el desarrollo del presente trabajo investigativo.

A los docentes de la Especialización en Epidemiología Alexandra Porras, Juan Carlos Aristizábal y al docente Fabio Ruiz Salazar del Programa de Psicología y quienes con su experiencia investigativa aportaron al mejoramiento de esta investigación.

A la ESE Hospital Comunal Malvinas, por permitirnos desarrollar la presente investigación y la información suministrada para la misma.

A los pacientes Hipertensos de la ESE Hospital Comunal Malvinas, por permitirnos acceder a sus hogares y brindarnos la confianza para el desarrollo del presente estudio.

\section{Referencias}

1. ORGANIZACIÓN MUNDIAL DE LA SALUD; Adherencia a los tratamientos a largo plazo: pruebas para la acción, 2004.

2. Alfonso G JP. Hipertensión Arterial en la Atención Primaria en Salud; editorial ciencias médicas, La Habana Cuba, 2009; p 169. 
R.F.S Revista Facultad de Salud

Enero-Junio de 2015;7(1): 32-38
Conocimientos, actitudes y prácticas del paciente hipertenso de la ciudad de Florencia, Colombia/César G. Gómez C. \& Cols.
3. Acosta, González, M. Conocimientos, creencias y prácticas en pacientes hipertensos, relacionados con su adherencia terapéutica; Revista Cubana Enfermería v. $21 N^{\circ} .3$ Ciudad de la Habana, sep.-dic. 2005.

4. COLOMBIA. MINISTERIO DE LA PROTECCIÓN SOCIAL, Guía de Atención de la Hipertensión Arterial. 2008.

5. COLOMBIA MINISTERIO DE LA PROTECCIÓN SOCIAL, Encuesta Nacional de Salud 2007, p 20.

6. Hernández Sampieri R, Fernández Collado C, Baptista Lucio P. Metodología de la investigación. Editorial Mc Graw Hill. Quinta edición. Cáp. 6 p. 2.

7. Buendía, JA; Actitudes, conocimientos y creencias del paciente Hipertenso sobre la medicación antihipertensiva; Biomédica, 2012; 32:578-84.
8. MINISTERIO DE SALUD PÚBLICA, Hipertensión Arterial, Guía para la Prevención, Diagnóstico y Tratamiento; La Habana 2008; p 18.

9. Huertas Paredes, JM. Actitudes Humanas, Actitudes Sociales. Universidad de Mayores de Experiencia Reciproca. Conferencia. (Madrid: 22 de octubre de 2007, p. 6).

10. Simal, Blanco F. Estudio Epidemiológico transversal de los factores de riesgo cardiovascular en la provincia de Valladolid: la Hipertensión Arterial y calidad de vida relacionada con la salud. Tesis doctoral, 2002. 